\title{
Structural MRI volumetric analysis in patients with organic amnesia, 1 : methods and comparative findings across diagnostic groups
}

\author{
A Colchester, D Kingsley, D Lasserson, B Kendall, F Bello, C Rush, T G Stevens, \\ G Goodman, G Heilpern, N Stanhope, M D Kopelman
}

Neuro-Media Group, KIMHS, Electrical Engineering Labs, University of Kent, Canterbury, Kent, CT2 7NT, UK

A Colchester

F Bello

C Rush

Department of Neurology, Guy's Campus, Guy's and St Thomas's Trust, London, SE1 9RT, UK A Colchester

Department of Radiology, The National Hospital, Queen Square, London, WC1N 3BG, UK

D Kingsley

B Kendall

University

Department of

Psychiatry and

Psychology, St

Thomas's Campus,

King's College London,

London, SE1 7EH, UK

D Lasserson

T Stevens

G Goodman

G Heilpern

N Stanhope

M D Kopelman

Correspondence to: Professor M D Kopelman michael.kopelman@kcl.ac.uk or

Professor AFC Colchester a.colchester@ukc.ac.uk

Received 21 July 1999 and in final form

18 December 2000

Accepted 5 January 2001

\begin{abstract}
Background-If they are to be replicable, MRI volume measurements require explicit definitions of structures and of criteria for delineating these structures on MRI. Previously published volumes in healthy subjects show considerable differences in measurements across different studies, including a fourfold variation in estimates of hippocampal volume. Previous neuroimaging reports in patients with Korsakoff syndrome have generally found widespread or non-specific change, whereas in patients with herpes encephalitis the extent of pathological involvement reported beyond the temporal lobes has varied.
\end{abstract}

Method-In the present study, a clear set of anatomical criteria and detailed MRI segmentation procedures were applied to measure whole brain, frontal and temporal lobe, and anterolateral and medial temporal volumes, as well as thalamic areas in patients with organic amnesia (from Korsakoff's syndrome, herpes encephalitis, and focal frontal lesions) as well as healthy controls.

Results-Patients with Korsakoff's syndrome showed decreased thalamic measurements but no significant changes in the medial temporal lobes, whereas patients with herpes encephalitis showed severe medial temporal but not thalamic atrophy. In the patients with known frontal lobe lesions, quantitative analysis on MRI showed reduced frontal lobe volume but no significant temporal lobe or thalamic atrophy.

Conclusion-Quantified MRI can be a useful technique with which to examine brain-cognitive relations, provided that detailed techniques are explicitly described. In particular, specific patterns of volume change can be found in vivo in patients with Korsakoff's syndrome and those with herpes encephalitis.

(F Neurol Neurosurg Psychiatry 2001;71:13-22)

Keywords: magnetic resonance imaging; volumetrics; amnesia; memory

Quantified radiological techniques can now be employed to measure crucial brain structures or lesions in neurological patients. Most published reports have focused on only a few disorders - for example, Alzheimer's dementia and epilepsy - and have measured a restricted range of anatomical structures. There have been relatively few reports in organic amnesia. ${ }^{1-4}$

Accurate quantification of areas or volumes of structures on MRI is difficult. There are no satisfactory and fully automated methods, and some degree of user interaction is required. Fully manual planimetric methods require the user to identify a structure and to trace its entire boundary but, generally, boundary placement in low resolution images has poor reproducibility. An alternative approach is stereology, ${ }^{5}$ in which quantification is reduced to counting points within a structure, but this is still dependent on the user's perception of where boundaries lie. Atlas based methods have also been developed, ${ }^{6}$ but these have difficulties where brain structures are grossly atrophied or distorted.

In practice, most published studies have employed planimetry or a variant: this requires that anatomical boundaries are clearly defined, using criteria identifiable on MRI. Variation in the criteria employed complicates comparisons across studies and is noticeable, for example, in studies reporting medial temporal volumes. ${ }^{7-10}$ Bronen and Cheung ${ }^{11}$ described particular difficulties in measuring the hippocampi.

Reports have also varied widely in the explicitness of their description of what was actually done. Given the neuroanatomical variation seen in normal subjects, ${ }^{11}$ clear rules are important and an element of pragmatism ("rules of thumb") is required in assessing boundaries when the normal anatomy is unclear or absent. Only a few reports ${ }^{48}$ give such details.

The accuracy of measuring a three dimensional neurological structure by a series of cross sectional areas, as occurs in MRI volumetric analysis, is also limited by the slice thickness and the gap between sequential scan slices. The variation in slice thickness has been substantial in measurements of hippocampal volume, ranging between $1.5 \mathrm{~mm}$ and $8.5 \mathrm{~mm} .^{7810} 12$ Large interslice distances reduce accuracy and provide a further obstacle to meaningful comparisons.

The repeatability or reliability of measurements varies within the same raters and between different raters, but interrater reliability has often not been reported. The validation of a segmentation technique (for delineating structures) cannot rely entirely on within rater measurements, especially when only a few slices, ${ }^{13}$ only one slice, ${ }^{7}$ or few subjects ${ }^{14}$ are examined. 
In this paper, we have carried out a volumetric analysis of MR scans from patients with organic amnesia. We employed an interactive computer analysis package,${ }^{15}$ which required the user to indicate a few points inside and outside a structure to be measured. From this, the computer selected the most appropriate boundaries from a set of automatically precomputed candidate boundaries. The user then edited these boundaries at a level of detail which he or she controlled. We measured a range of neurological structures, applying an explicit set of anatomical criteria and segmentation (demarcation) rules: these have been documented in sufficient operational detail that our measurements could be repeated by others. We used all the available slices in our data acquisition to improve the accuracy of our volume measurements. Structural volume findings are analysed in terms of (1) measurement repeatability in healthy subjects; and (2) comparisons between findings in Korsakoff, herpes encephalitis, and focal frontal lesion groups. Correlations with neuropsychological impairments are reported in the companion paper. ${ }^{16}$

\section{Methods}

MR ACQUISITION PROTOCOL

The first 17 subjects were scanned on a Siemens Magnetom SP 63 1.5 Tesla scanner and the next 19 subjects on a General Electric 1.5 Tesla Signa scanner (because of delays in installation of the second scanner). All subjects had a conventional proton density and T2 weighted sequence for basic visual analysis, using $5 \mathrm{~mm}$ thick slices with an interslice gap of $2.5 \mathrm{~mm}$. The field of view (FOV) was $250 \mathrm{~mm}$ (Magnetom) and $240 \mathrm{~mm}$ (Signa). In addition, separate $\mathrm{T} 1$ and $\mathrm{T} 2{ }^{\star}$ weighted gradient echo three dimensional data sets were obtained in the sagittal plane using 64 partitions with the Magnetom (effective slice thickness $2.34 \mathrm{~mm}$ ) and 128 partitions with the Signa scanner (effective slice thickness $1.20 \mathrm{~mm}$ ). The matrix size was $256 \times 256$ on both scanners with FOVs as above. This gave a voxel volume of $2.23 \mathrm{~mm}^{3}$ on the Magnetom $(0.98 \times 0.98 \times 2.34 \mathrm{~mm})$ and $1.05 \mathrm{~mm}^{3}$ on the Signa scanner $(0.94 \times 0.94 \times 1.20 \mathrm{~mm})$. Patients from each diagnostic group were fairly evenly distributed between the two scanners. However, hippocampal measurements could be made only from images from the second scanner.

INTERACTIVE COMPUTER ANALYSIS PACKAGE

A graphics workstation (HP 735) was used to carry out segmentation (delineation) of brain structures across sequential MR slices, using locally developed software. ${ }^{15}$ An application specific graphical user interface (GUI) incorporated all the necessary visualisation, manipulation, and storage or retrieval functions. The segmentation tools included a multislice two dimensional hierarchical segmentation program, ${ }^{15}$ a standard two dimensional polyline tool for drawing a sequence of connected straight lines, and a three dimensional plane cutting tool.

The hierarchical segmentation tool was based on an agglomerative clustering algorithm which worked by grouping pixels for which the edge strength was low locally, according to local grey level differences and edge stability in scale space. ${ }^{15}$ Boundaries which corresponded to ridges of locally high edge strength were established between pixels that belonged to different clusters. The resulting regions were then subjected to the same clustering process to form larger regions and this process was repeated until the regions were grouped hierarchically into the whole image. Computation was fully automatic and was done at the beginning of a session. The results were not shown on the monitor at this stage. Once the hierarchy had been computed, the user marked points inside and outside the required anatomical structure by using the mouse buttons. The computer then searched the hierarchy from the top down to find the largest structure which contained all the inside points and none of the outside points. If there was no such structure, the hierarchy was amended to create a valid structure complying with the constraints specified by the user.

A polyline was defined by the user providing a set of control points that could be edited interactively. It was not necessary for a polyline to form a closed structure, but a facility was available to obtain closed structures from an open ended polyline definition. The plane cutting tool allowed the user to generate a two or three dimensional plane from a series of interactively specified points that could be edited simultaneously. Once a plane had been defined, it could be used to define part of the boundary of structures which were then processed further.

Integration of the hierarchical segmentation, polyline, and plane cutting tools was controlled interactively by the user so that the structures of interest could be segmented according to specific criteria, as described below.

DEFINITION OF BOUNDARIES OF NEUROANATOMICAL STRUCTURES

Intracranial vault volume

The intracranial space above the tentorium was segmented along the interface between CSF and dura using the $\mathrm{T} 2{ }^{\star}$ three dimensional data set. Laterally, the tentorium is easy to identify, separating the cerebellum from the cerebral hemisphere. Medially, the cerebral peduncles were cut off by drawing a line from the estimated position of the anterior free margin of the tentorium to the posterior clinoid processes. Where the clinoids were not visible, the posterior margin of the floor of the anterior cranial fossa (tuberculum sellae) was used as the inferomedial marker of the tentorial attachment to the skull base.

\section{Whole brain (supratentorial)}

T2 weighted scans in the sagittal plane were used to give good contrast between the cortical surface and CSF which was excluded from measurements of brain volume. As far as possible, all sulci were outlined, and vessels, the falx, and infratentorial structures were excluded from segmentation. 

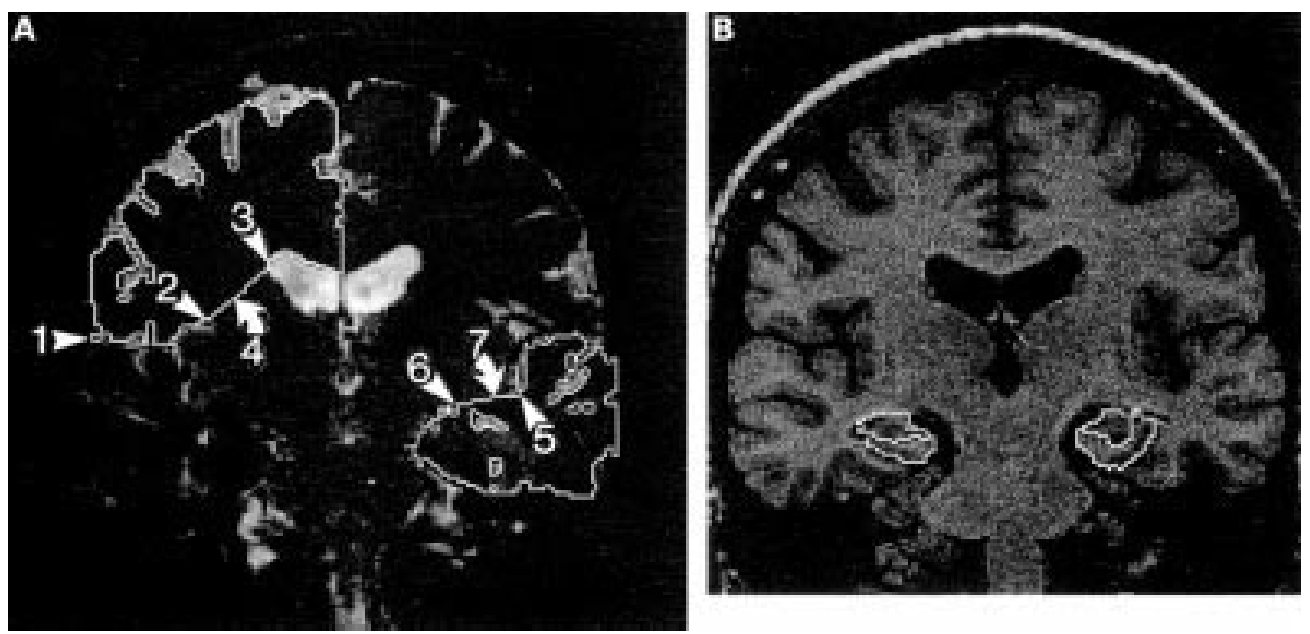

Figure 1 Mid-coronal MRI slice (slice 128): (A) T2* slice showing frontal (upper) and temporal lobe (lower) segmentations. Line 4 was drawn from the superior fundus of the circular sulcus to the superolateral tip of the lateral ventricle (frontal lobe), and line 7 from the inferior fundus of the circular sulcus (5) to the most lateral invagination of the choroidal fissure (6). (B) T1 slice indicating medial temporal (combined parahippocampal and hippocampus) segmentation demarcating the hippocampal/parahippocampal boundary.

Frontal lobe

The superior, medial, and lateral surfaces are bounded by CSF, and were defined in the whole brain segmentation. Additional boundaries were defined on serial coronal sections starting at the front and working backwards. In the anterior sections, the inferior surface is bounded by subarachnoid space. In progressively more posterior sections, deep structures including the anterior basal ganglia start to appear as forward invaginations into the frontal lobe, and a little further back the temporal stem becomes visible. Before the temporal stem was reached, the deep structures above the optic chiasm were not excluded from the frontal lobe as the volume contributed by these structures is small, and it is difficult otherwise to define a plane clearly. Once the temporal stem was present (fig $1 \mathrm{~A}$ ), the segmentation involved drawing a polyline from the inferolateral edge of the frontal operculum (1) into the sylvian fissure to the insula, then upwards into the fundus of the circular sulcus (2). From there a straight line was drawn to the superolateral tip of the lateral ventricle (3). The roof of the lateral ventricle was followed to the midline. This procedure was continued to the posterior limits of the frontal lobe.

The posterior boundary was formed by a plane defined by three points:

Point 1: the superomedial limit of the central sulcus-The central sulcus was recognised on axial slices as the most prominent sulcus running obliquely backwards and reaching the interhemispheric fissure. ${ }^{17}$ The point landmark of its superomedial limit was defined by extrapolation from the gyral convexities on either side by viewing the MR slices axially to establish the anteroposterior coordinates of the point, and by viewing a coronal section to judge the superoinferior and the mediolateral position ( $\mathrm{x}, \mathrm{y}, \mathrm{z}$ coordinates)

Point 2: the posterior commissure-The posterior commissure (PC) in the midline, lying just above the superior colliculus, was the second point landmark. If not clearly seen on
$\mathrm{T} 2^{\star}$, its position could be located in relation to the surrounding structures.

Point 3: the inferolateral limit of the central sulcus-The central sulcus was followed downwards through its full extent. At its tip, a line was drawn perpendicular from its inferior limit to the sylvian fissure, and their junction was the third landmark.

\section{Temporal lobe}

The anterior, lateral, and inferior boundaries were defined in the whole brain segmentation. The medial boundary was followed up into the suprasellar cistern and into the choroidal fissure.

The superior boundary of the temporal lobe was segmented by viewing coronal slices sequentially from front to back. Anteriorly, the temporal pole was fully isolated by CSF. However, once the temporal stem was encountered, the superomedial margin was defined by a line from the inferior fundus of the circular sulcus of the insula (5) to the most lateral invagination of the choroidal fissure (6 in fig $1 \mathrm{~A}$ ). Posteriorly, the temporal stem disappears, although the sylvian fissure is still present. At this point, the superomedial boundary was drawn from the medial limit of the sylvian fissure (laterally) to the tip of the trigone of the lateral ventricle, and then to the choroidal fissure (medially).

The posterior boundary was formed by a plane defined by three points. Points 1 and 2 (the superomedial limit of the central sulcus and the posterior commissure in the midline) were as above. The third point for the temporal lobe was an approximation of the preoccipital notch. On the appropriate axial slice (fig 2) the line joining the AC-PC line was established by identifying the anterior commissure (AC) and the point on the axial slice closest to the position of the PC. The AC-PC line was extended anteriorly and posteriorly to the surface of the brain (a), and a point was identified three quarters of the way back along this line, from which a line was drawn perpendicular to the AC-PC line. Its intersection with the 


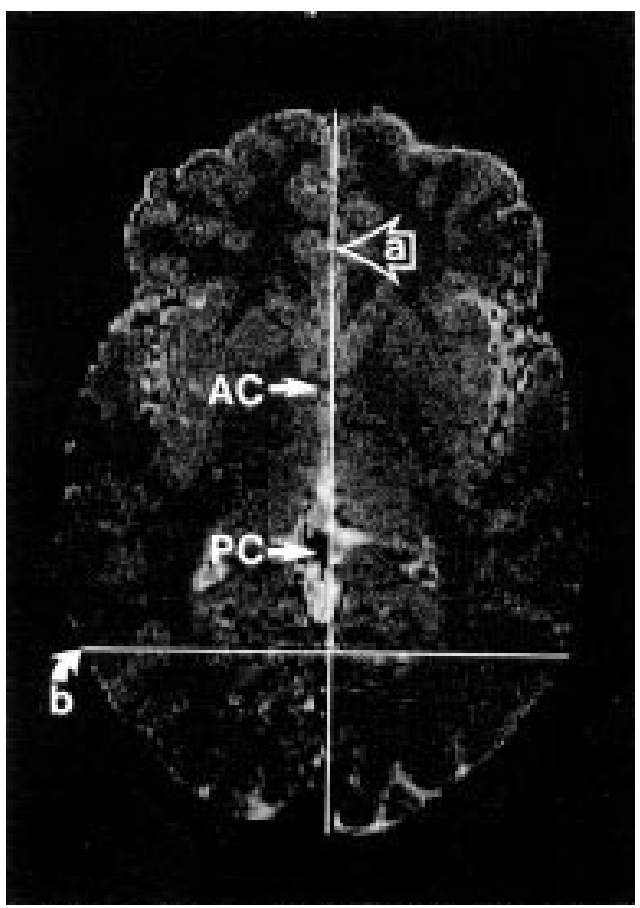

Figure 2 How the position of the preoccipital notch was approximated on an axial MRI slice for determination of the posterior boundary of the temporal lobe. The slice closest to the AC-PC line was selected. The median sagittal line (a) (our approximation of the AC-PC line) was extended to the surface of the brain and a point was identified three quarters of the way back along this line. From this point, a line was drawn at right angles, and its intersection with the arachnoid surface of the brain (b) was taken as an approximation of the preoccipital notch

arachnoid surface of the brain defined the third point landmark (b).

\section{Medial temporal lobe structures}

Separate segmentations were carried out on coronal sections to measure hippocampal volume and "medial temporal" (combined hippocampal and parahippocampal) volume (fig $1 \mathrm{~B}$ ). Our boundary definitions for the hippocampus were closely similar to those described by Mori et $a l,{ }^{12}$ except that we included the subiculum as part of the hippocampus. Anteriorly, the alveolar covering of the hippocampus provided a border with the amygdala. The posterior limit was the coronal slice in which the fornix clearly emerged from the fimbria of the hippocampus, just anterior to the splenium of the corpus callosum. These margins were checked in sagittal and axial sections. The "medial temporal" measurement employed the same anterior and posterior margins but, in the coronal plane, segmentations were taken from the subiculum around the cortical surface of the parahippocampal gyrus, and then deep into the collateral (rhinal) sulcus until it met the inferolateral point of the hippocampus.

\section{Thalamus}

The lateral thalamic boundary was not clearly delineated from the internal capsule on $\mathrm{T} 1$ or T2 weighted images. Consequently, thalamic measurements were made on axial proton density (PD) images where the lateral boundary was clear. Because only a limited number of
PD axial slices were available, we made a thalamic area measurement at the slice giving the maximum thalamic area. The volume of the third ventricle was obtained by segmenting its outline in the sagittal slices where it was visible. Its posterior margin was drawn from the superior colliculus along the inferior margin of the internal cerebral veins. We calculated, thereby, a "thalamic index" (the ratio of thalamic area : third ventricle volume) as an alternative method of estimating thalamic atrophy.

\section{Lesion volume}

Where there was a discrete lesion (or lesions), usually in the temporal or frontal lobes, this was segmented in sagittal views, and its volume was subtracted from that of the respective lobe.

\section{Mammillary bodies}

We considered that valid volumetric measurements of these could not be determined, because of the problem of partial volume artefact across the few sections transecting this very small structure. Consequently, ratings on MRI films (checked against three dimensional images on the GUI viewing screen) were made by a consultant neuroradiologist (DK), blind to each subject's clinical diagnosis. Ratings were classified along a three point scale: normal, visible but small, and not visible.

\section{SUBJECTS}

From 20 healthy subjects, who had taken part in cognitive studies ${ }^{18}$ and who matched our total group of patients in terms of mean age $(F(6,57)=1.35 \mathrm{NS}), 10$ healthy volunteers participated in the MR studies. These 10 volunteers were somewhat younger (mean 39.1 (SD 13.5) years than the larger group of controls (mean 45.9 (SD 17.3 years)), but otherwise they were matched in terms of current IQ, memory indexes, and scores on frontal/ executive tests.

Comparisons were made between findings of different lesion groups. Patients with focal frontal lesions were included to establish that such pathology could indeed be quantified in vivo with a view to investigating the relation between frontal volume and cognitive function. There were six patients with focal frontal pathology, selected on CT evidence of lesions which sappeared confined to the frontal lobes, and which resulted from a haematoma, infarcts, craniotomy, or (in one case) leucotomy scars from a bilateral operation 18 years earlier. Three of these patients had right sided lesions, one a left sided lesion, and two had bilateral lesions. Secondly, patients with herpes encephalitis were included (1) to quantify the temporal lobe changes, and (2) to examine the extent of pathological change elsewhere, which has varied in previous MR reports on such patients. ${ }^{49}$ There were nine patients selected on the basis of a history of viral encephalitis, consistent with herpes simplex, serologically proved in most cases, and with CT evidence of severe temporal lobe pathology. Thirdly, a group of patients with Korsakoff's syndrome was examined: on the basis of pathological findings, diencephalic changes would be pre- 
Table 1 Background cognitive scores: means (SD) (tests as described in Kopelman et al ${ }^{18}$ )

\begin{tabular}{lllll}
\hline & $\begin{array}{l}\text { Healthy controls } \\
(n=10)\end{array}$ & $\begin{array}{l}\text { Korsakoff patients } \\
(n=11)\end{array}$ & $\begin{array}{l}\text { Herpes encephalitis } \\
\text { patients }(n=9)\end{array}$ & $\begin{array}{l}\text { Focal frontal lesion } \\
\text { patients }(n=6)\end{array}$ \\
\hline $\begin{array}{l}\text { Background intelligence tests: } \\
\quad \text { NART-R IQ }\end{array}$ & $106.1(14.9)$ & $108.9(11.9)$ & $103.3(11.6)$ & $107.5(14.0)$ \\
$\quad \begin{array}{l}\text { WAIS-R FS IQ } \\
\text { Memory tests: }\end{array}$ & $104.0(18.3)$ & $99.5(14.5)$ & $96.9(12.9)$ & $102.3(21.7)$ \\
$\quad$ WMS-R GMQ & $103.3(16.1)$ & $69.5(20.4)$ & $66.6(14.7)$ & $80.2(19.6)$ \\
$\quad \begin{array}{l}\text { WMS-R DMQ† } \\
\text { Executive tests: }\end{array}$ & $103.3(20.3)$ & $61.0(16.0)$ & $65.6(13.3)$ & $76.5(16.6)$ \\
$\quad$ FAS verbal fluency & $44.2(15.0)$ & $31.0(14.3)$ & $36.3(13.7)$ & $29.7(15.0)$ \\
$\quad$ Card sorting categories & $5.1(1.4)$ & $3.8(1.9)$ & $4.7(1.6)$ & $4.0(1.7)$ \\
$\quad \begin{array}{l}\text { Card sorting \% perseverations } \\
\text { Cognitive estimates }\end{array}$ & $9.7(9.7)$ & $31.7(27.6)$ & $26.6(29.2)$ & $23.3(14.3)$ \\
\hline
\end{tabular}

${ }^{\star}$ General memory index (or quotient) from Wechsler memory scale-revised.

†Delayed memory index (or quotient) from Wechsler memory scale-revised.

dicted but, to date, it has proved very difficult to demonstrate these in vivo with any precision or consistency on neuroimaging. ${ }^{13}{ }^{20-22}$ There were 11 patients with a clinical diagnosis of Korsakoff's syndrome, of whom 10 had a clearly documented history of Wernicke's features. None of these patients showed focal cortical pathology on naked eye inspection of their scans.

Table 1 gives background cognitive data for these subject groups, demonstrating severe impairment in the patient groups on the general $(F(1,34)=8.70, \mathrm{p}<0.0002)$ and delayed $(F(1,34)=12.97, \mathrm{p}<0.0001)$ memory indexes, and a more moderate impairment on executive tests. ${ }^{19}$

ASSESSMENT OF MEASUREMENT REPEATABILITY Comparisons were made between two assessors for control whole brain and hippocampal measurements in terms of interrater reliability coefficients (the intraclass correlation ${ }^{23}$ is a Pearson interrater correlation corrected for differences in the within rater (intraclass) variance ${ }^{23}$ and mean error measurements. Intraclass reliability correlations were also examined for hippocampal values in a subgroup of 12 patients and in a combined group of 22 patients and controls.

HYPOTHESES

It was hypothesised that the patient groups would show regional volume loss consistent with their clinical diagnoses-medial temporal lobe volume in the patients with herpes encephalitis, thalamic volume in the patients with Korsakoff's syndrome, and frontal volume in the frontal group. The extent of concomitant involvement of other structures-thalamic and frontal in the herpes group, frontal and medial temporal in the Korsakoff group, and thalamic/ medial temporal in memory disordered frontal patients - remains controversial from previous investigations, and was also a matter of interest: the null hypothesis was that these structures would be unaffected.

\section{Results}

ASSESSMENT OF MEASUREMENT REPEATABILITY Whole brain volume

Comparison was made between segmentations in the 10 healthy controls by an assessor at Stonybrook Hospital, NY (under the direction of Dr Lynn De Lisi) and by DL. The mean error between them was $2.4 \%$ (range 0.1 to $4.7 \%)$. The intraclass reliability correlation was $0.96(p<0.0001)$

\section{Hippocampal volumes}

Volumetric measures by two United Kingdom assessors were compared for the left and right hippocampus across 10 healthy control subjects. The mean difference between assessors was $8.0 \%$, and their intraclass reliability correlation across the 20 measurements (10 left; 10 right) was $0.79(\mathrm{p}<0.001)$. In a subgroup of 12 patients of various aetiologies, the intraclass correlation was $0.81(\mathrm{p}<0.001)$, and in the combined subset of 22 controls and patients, interrater reliability was $0.85(\mathrm{p}<0.001)$.

\section{Interscanner comparisons}

Because the subjects had been scanned in two different machines, we carried out a two way analysis of variance (ANOVA), examining the effects of scanner and diagnostic group, across each of the outcome measures. None of the effects of scanner or scanner by diagnostic group was significant. In the total subject group, mean intracranial volume was 1.2511 (SD 0.014) on scanner 1 versus 1.2571 (SD 0.022 ) on scanner 2 , and among the patients mean brain volume was 0.9411 (SD 0.111) versus 0.8901 (SD 0.104). Findings within the individual patient groups were consistent with this-for example, mean brain volume was 0.8741 (SD 0.106) in five patients with herpes encephalitis on scanner 1 versus 0.8721 (SD 0.112 ) in four patients with herpes encephalitis on scanner 2 .

STRUCTURAL VOLUME CHANGES AND DIAGNOSTIC CATEGORY

Brain volumes were evaluated for both actual observed volumes and volumes normalised for intracranial volume using the formula of Jack et $a l .{ }^{24}$ This formula corrects an observed regional brain volume according to the difference between that subject's intracranial volume and the control mean, and the regression of regional brain volumes against intracranial volumes in controls. However, we found that it was much more legitimate in correcting the volumes of large structures than of small ones, in terms of the percentage of variance accounted for by the regression equation and its statistical significance. For example, regressing left frontal volume against intracranial volume 


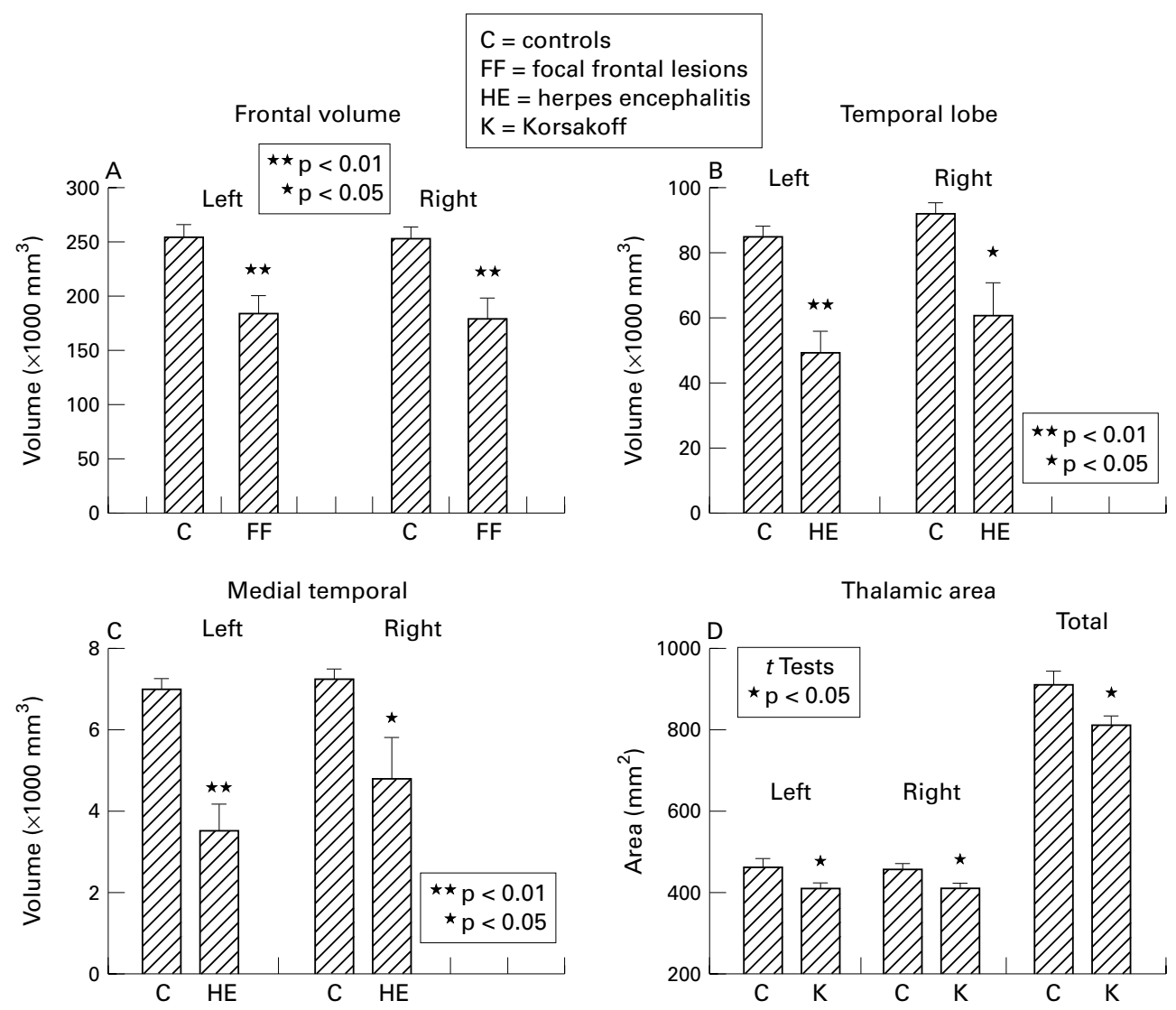

Figure 3 Comparison of mean (SEM) measurements across: (A) frontal lobe volume: controls $v$ focal frontal lesion group; (B) temporal lobe volume: controls v herpes encephalitis; (C) medial temporal lobe:controls v herpes encephalitis; (D) thalamic area: controls $v$ Korsakoff group.

accounted for $63.7 \%$ of left frontal variance in controls $(\mathrm{p}<0.01)$, but the equivalent regression accounted for only $0.01 \%$ of left medial temporal variance (non-significant). We report the findings for actual volumes, but statistical analyses were performed on both actual and corrected volumes in all instances, and only minor differences were obtained.

Frontal lobe volumes in vivo across the four subject groups showed significant differences (left: $F(3, \quad 30)=11.02, \quad \mathrm{p}<0.0001$; right: $F=11.03, \mathrm{p}<0.0001)$. Student-Newman-Keuls post hoc tests showed significant differences between each of the patient groups and healthy controls. Figure 3 A shows the left and right mean frontal volumes in healthy controls and the focal frontal group.

Temporal lobe volume also differed significantly across the diagnostic groups $(F(3,30)=12.58, \mathrm{p}<0.0001)$. On the post hoc tests, the herpes group differed significantly from the healthy controls, patients with Korsakoff's syndrome, and patients with focal frontal lesions $(p<0.01$ in all cases). Figure 3 B compares mean left and right temporal lobe volumes in the control and herpes groups. Further analysis of the pattern of temporal lobe atrophy is more meaningful after subdividing the temporal lobe into anterolateral and medial structures.

Anterolateral temporal lobe volume was determined by subtracting medial temporal (hippocampal and parahippocampal) volume from whole temporal lobe volume for each hemisphere. For both left and right anterolateral temporal volume, the four groups differed significantly (left: $F(3,30)=10.03, \mathrm{p}<0.0001$; right: $F=4.55, \mathrm{p}<0.01)$. On post hoc tests, the herpes group differed significantly from the other three groups (left: $p<0.01$; right: $p<0.05$ ) but the controls, Korsakoff, and focal frontal groups did not differ significantly from one another.

Medial temporal volume differed significantly across the subject groups (left: $F(3$, $31)=19.17$, p<0.0001; right $F=4.83$, p<0.01). On the post hoc tests, the herpes group differed significantly from the healthy controls, Korsakoff group, and focal frontal group (left: $\mathrm{p}<0.01$; right: $\mathrm{p}<0.05)$. There were no other statistically significant differences between the groups. Figure $3 \mathrm{C}$ shows a comparison of mean left and right medial temporal volumes in the herpes and control groups.

Parahippocampal volume was determined by subtracting hippocampal from medial temporal volume. Figure $4 \mathrm{~A}$ shows the mean total (left and right) parahippocampal volume across the four groups, which differed significantly $(F(3,16)=6.19, \mathrm{p}<0.0005)$, and on the post hoc tests, the herpes group differed significantly $(p<0.05)$ from the other three groups. For left parahippocampal volume, the groups also differed significantly $(F(3$, $16)=3.58, \mathrm{p}<0.05)$ with the herpes group showing a significantly $(p<0.05)$ smaller volume than the controls; but for right parahippocampal volume, the groups did not differ 

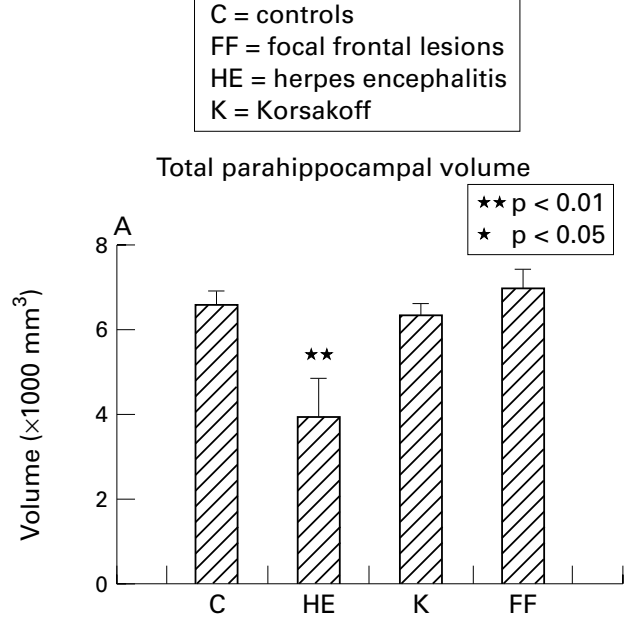

Total hippocampal volume

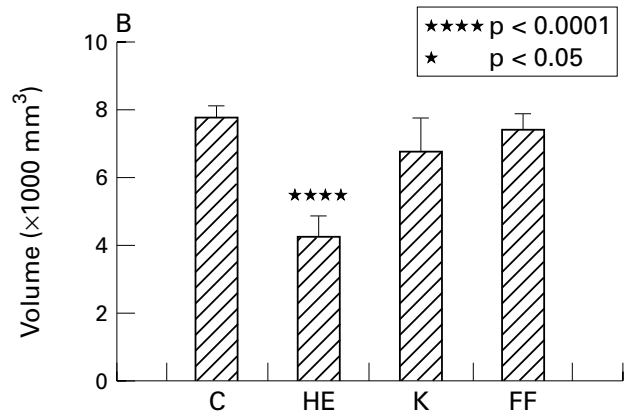

Total thalamic area

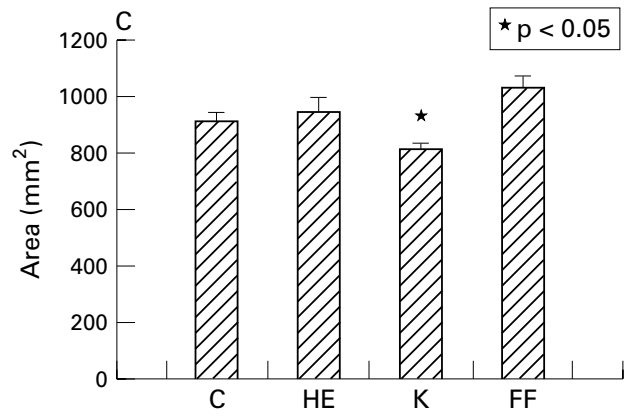

Figure 4 Comparison of mean (SEM) medial temporal and thalamic measurements across all four groups (controls, herpes, Korsakoff and focal frontal): (A) total (left and right) parahippocampal volume; (B) total (left+right) hippocampal volume; $(C)$ total (left+right) thalamic area. The figure shows significant $t$ test findings compared with healthy controls. For ANOVAS and post hoc tests see text.

significantly, although there was a clear trend for the herpes group to show the smallest volumes.

Figure $4 \mathrm{~B}$ shows mean total (left and right) hippocampal volumes across the four groups, which differed significantly $(F(3,16)=9.41$, $\mathrm{p}<0.001)$ and, on post hoc tests, the herpes group differed significantly $(\mathrm{p}<0.05)$ from the

Table 2 Mammillary bodies

\begin{tabular}{lllll}
\hline Group & Normal & Small & Not visible & $\%$ Abnormal \\
\hline Controls & 10 & 0 & 0 & 0 \\
Focal frontal & 4 & 1 (=bilateral vascular) & 0 & 20 \\
Herpes encephalitis & 6 & 3 & 0 & 33 \\
Korsakoff & 4 & 5 & 2 & 64
\end{tabular}

Atrophy $v$ no atrophy $\chi^{2}=10.12 ; \mathrm{p}<0.02$. control, Korsakoff, and focal frontal groups. The groups also differed significantly for left $(F$ $(3,16)=3.20, \mathrm{p}=0.05)$ and right $(F=5.67$, $\mathrm{p}<0.01)$ hippocampal volume. On post hoc tests, the herpes group differed significantly from the other three groups for right $(p<0.05)$ but not left sided volume. However, left hippocampal volume was significantly smaller in the herpes group than controls on a $t$ test $(t=2.99, \mathrm{p}<0.02)$.

Figure $4 \mathrm{C}$ compares total thalamic area across the four groups, and figure $3 \mathrm{D}$ compares patients with Korsakoff's syndrome and controls for the left and right thalami. The groups differed significantly for total area $(F(3$, $32)=5.10, \mathrm{p}<0.005)$. The Korsakoff group differed significantly from the focal frontal group ( $\mathrm{p}<0.05)$, and from healthy controls on $t$ tests (total area: $t=2.61$; left: $t=2.08$; right: $t=2.56 ; \mathrm{p} \leqslant 0.05$ in each case) and from the herpes group for total and right thalamic area $(t=2.40$ and $2.44, \mathrm{p}<0.05)$ and at near significance for the left thalamus $(t=2.01, \mathrm{p}<0.06)$. Table 2 shows the findings for ratings of mammillary body atrophy. Sixty four per cent of the patients with Korsakoff's syndrome showed atrophy versus $33 \%$ or less in the other groups $(\chi 2=10.12, \mathrm{p}<0.02)$.

In summary, the patients with herpes encephalitis showed significant atrophy of the medial temporal lobe structures, in the presence of normal thalamic areas and a much lower incidence of mammillary body atrophy than the Korsakoff group. By contrast, the patients with Korsakoff's syndrome showed significant atrophy of the thalami and mammillary bodies, but not of medial temporal structures (hippocampi, parahippocampal gyri, or the two measures combined). All three patient groups showed evidence of frontal lobe loss of volume, but the focal frontal lesion group did not show any atrophy in the temporal lobes or thalami.

\section{Discussion}

SOURCES OF ERROR AND MEASUREMENT REPEATABILITY

Table 3 shows that published volumes of brain structures in healthy subjects vary widely. Data are presented as actual volumes, normalised, ${ }^{24}$ or as a percentage of intracranial volumes, as in the original publications, except for our own (KCL/UKC) results, which are presented in all three ways. Hippocampal volumes vary particularly widely: total (left plus right) hippocampal volume ranged from $2519^{13}$ and 2614 $\mathrm{mm}^{323}$ to $10166^{25}$ and $10360 \mathrm{~mm}^{326}$ This fourfold variation is not accounted for simply by age or sex differences. Given the lack of published details about definitions of boundaries and their application during segmentation in many studies, this wide variability is likely to reflect differences in measurement technique. It also demonstrates the limitations of simply publishing reliability coefficients in the absence of these explicit details.

For whole brain volume, our interrater error (mean $2.4 \%$ ) and reliability coefficient (intraclass correlation $=0.96$ ) were very satisfactory. Interrater reliability was less accurate for the 

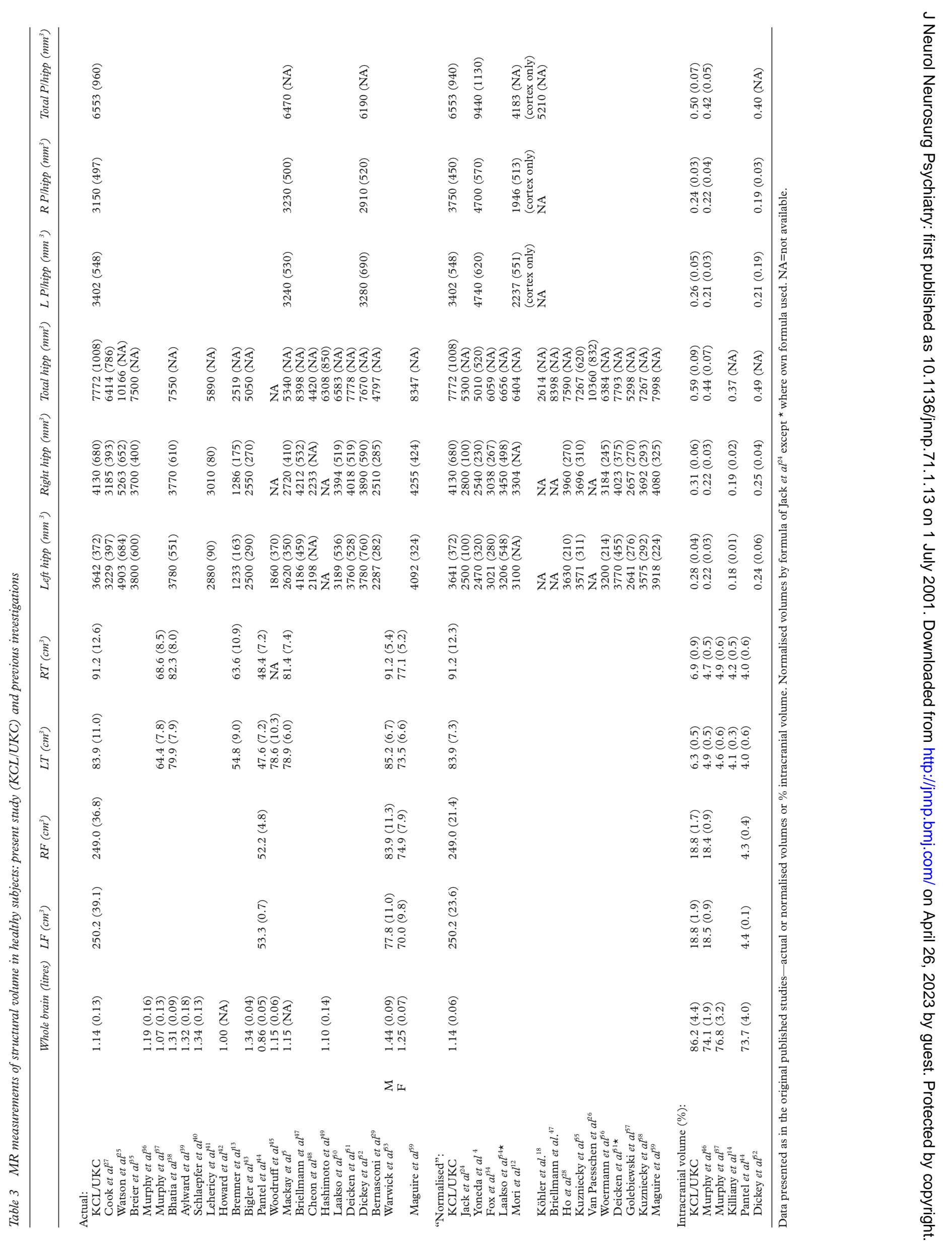
hippocampus. Our mean hippocampal error in controls $(8.0 \%)$ compares with $7 \%,{ }^{27} 5.1 \%,{ }^{28}$ and $4.1 \%,{ }^{29}$ and an intrarater error of $6.7 \% .^{30}$ However, our hippocampal intraclass correlation $(r=0.79)$ is in line with other studies-for example, $0.78^{13}$ and $0.82 .{ }^{23}$ Interrater reliability in patients has seldom been reported before: in the present study, the intraclass correlation for hippocampi was 0.81 , and in combined patients and controls it was 0.85 .

\section{STRUCTURAL VOLUME CHANGES AND DIAGNOSTIC} CATEGORY

The focal frontal group showed statistically significant losses of volume across left, right, and total frontal lobe measurements, but did not show any evidence of loss of volume in the thalami or on our measures of medial temporal, anterolateral temporal, and total temporal lobe volume. In previous neuropsychological studies, ${ }^{31}$ similar patients have been reported but without any MR quantification: such measurements permit in vivo correlation of loss of frontal tissue with cognitive performance. ${ }^{16}$

The patients with Korsakoff's syndrome showed significant atrophy of the left and right thalami, mammillary bodies, and frontal lobes. All the patients with Korsakoff's syndrome showed evidence of thalamic atrophy on at least one of our measures. Sixty four per cent of the Korsakoff group in the study also showed mammillary body atrophy. By contrast, this group did not differ significantly from healthy controls for anterolateral, medial temporal, parahippocampal, or hippocampal volume. Although this in vivo finding of thalamic and mammillary body atrophy is consistent with results obtained from neuropathological studies, conflict remains about which thalamic sites and pathways are critical for memory, ${ }^{32}{ }^{33}$ and previous neuroimaging studies have generally reported either widespread or non-specific changes only. ${ }^{20-22}$ Jernigan et $a l^{1}$ found significant atrophy in eight patients with Korsakoff's syndrome within a block of tissue labelled "diencephalic grey matter", which encompassed the thalami, mammillary bodies, other hypothalamic grey matter, and the septal nuclei. The present findings indicate that focal changes can be found in the thalami (and the mamillary bodies) taken in isolation.

Our patients with herpes encephalitis showed a highly significant anterolateral and medial temporal loss of volume. Consistent with this, they also showed significantly reduced parahippocampal and hippocampal volumes. By contrast, their thalamic readings did not differ significantly from (and tended to be slightly larger than) those of the control group. Unlike the Korsakoff group, the mammillary bodies were visible in all our patients with herpes encephalitis, although three out of nine $(33 \%)$ showed a moderate degree of atrophy. The herpes group also showed evidence of frontal lobe atrophy. To our knowledge, the only comparable study of patients with herpes encephalitis is that by Yoneda $e t a l^{4}$ who examined five patients and reported significant atrophy in the hippocampal formation and parahippocampal gyri. There was a lesser degree of atrophy in the amygdaloid body and anterior temporal neocortex, but these findings were not statistically significant in this small group of patients. These authors did not make measurements elsewhere. In a non-quantitative study, Kapur et $a l^{19}$ reported damage in the anterior and inferior temporal lobe gyri, the fornix, the mammillary bodies, the thalamic nuclei (in $50 \%$ of cases), the frontal lobes, and the striatum, as well as "limited involvement" of the cingulate gyrus, the parietal and occipital lobes. This study was non-quantitative, and consequently comparisons are difficult. However, neither visual inspection nor quantification suggested any damage to the thalamic nuclei in our patients, and the mammillary bodies were affected in only a minority of cases, and then to only a minor degree.

\section{Conclusions}

We have emphasised the importance of clear definitions of brain structures and of operational procedures in carrying out MR volumetric measurements, arguing that reliability and validity measurements are only useful in this context and pointing to the wide variation of findings in healthy subjects in previous studies. We obtained mean error and interrater reliability coefficients for whole brain which were very satisfactory, and hippocampal values which were comparable with other studies. We found that patients with Korsakoff's syndrome showed thalamic but not medial temporal atrophy on these in vivo measurements, whereas patients with herpes encephalitis manifested medial temporal but not thalamic atrophy (a double dissociation). Patients with known frontal lobe pathology showed in vivo frontal volume reduction on quantification, but did not show atrophy elsewhere (a single dissociation). Our criteria and techniques can be developed further to examine other, specific brain regions.

This research was funded by a Wellcome Trust project grant to MDK and BK and by European Union grant 950845 (Biomorph) grant to $\mathrm{AC}$ and colleagues.

1 Jernigan T, Schaffer K, Butters N, et al. Magnetic resonance maging of alcoholic Korsakoff patients. Neuropsychopharmacology 1991;4:175-86.

2 Press GA, Amaral DG, Squire LR. The neurology of memory: a high-resolution magnetic resonance imaging protocol reveals reduced size of the hippocampal formation in amnesic patients. Nature 1989;341:54-7.

3 Squire LR, Amaral DG, Press GA. Magnetic resonance imaging of the hippocampal formation and mammillary nuclei distinguish medial temporal lobe and diencephalic amnesia. f Neurosci 1990;10:3106-17.

4 Yoneda Y, Mori E, Yamashita H, et al. MRI volumetry of medial temporal lobe structures in amnesia following medial temporal lobe structures in amnesia following
herpes simplex encephalitis. Eur Neurol 1994;34:243-52.

5 Mackay CE, Roberts N, Mayes AR, et al. An exploratory study of the relationship between face recognition memory and the volume of medial temporal lobe structures in healthy young males. Behav Neurol 1998;11:3-20.

6 Ashburner J, Hutton C, Frackowiak R, et al. Identifying global anatomical differences: deformation-based morphometry. Hum Brain Mapp 1998;6:348-57.

7 Deweer B, Lehéricy S, Pillon B, et al. Memory disorders in probable Alzheimer's disease: the role of hippocampal atrophy as shown with MRI. I Neurol Neurosurg Psychiatry 1995;58:590-7.

8 Golomb J, Kuger A, de Leon MJ, et al. Hippocampal formation size in normal human aging: a correlate of delayed sec-

Jack CR. MRI-based hippocampal volume measurements in Jack CR. MRI-based hippocampal volume

10 Wilson RS, Sullivan M, de Toledo-Morrell L, et al. Association of memory and cognition in Alzheimer's disease with volumetric estimates of temporal lobe disease with volumetric estimates of te
structures. Neuropsychology 1996;10:459-63. 
11 Bronen RA, Cheung G. Relationship of hippocampus and amygdala to coronal MRI landmarks. Magn Reson Imaging 1991:9:449-57.

12 Mori E, Yoneda Y, Yamashita H, et al. Medial temporal structures relate to memory impairment in Alzheimer's disease: an MRI volumetric study. $\mathcal{F}$ Neurol Neurosurg Psychiatry 1997;63:214-21.

13 Bremner JD, Randall P, Scott TM, et al. MRI-based measurement of hippocampal volume in patients with combat-related posttraumatic stress disorder. Am f Psychiatry 1995;152:973-81.

14 Killiany RJ, Moss MB, Albert MS, et al. Temporal lobe regions on magnetic resonance imaging identify patients with early Alzheimer's disease. Arch Neurol 1993;50:94954.

15 Griffin LD, Colchester ACF, Roell SA, et al. () Hierarchical segmentation satisfying constraints. In: Hancock ER, ed. Proceedings of the British Machine Vision Conference. Sheffield: BMVA Press, 1994:135-44.

16 Kopelman MD, Colchester A, Lasserson D, et al. Structural MRI volumetric analysis in patients with organic amnesia. 2: correlations with anterograde memory and executive tests in 40 patients. $f$ Neurol Neurosurg Psychiatry 2001;70 $23-8$.

17 Steinmetz H, Furst G, Freund H-J. Cerebral cortical localisation: application and validation of a proportional grid system in MR imaging. 7 Comput Assist Tomogr 1991;13:10-19

18 Kopelman MD, Stanhope N, Kingsley D. Retrograde amnesia in patients with diencephalic, temporal lobe, or frontal lesions. Neuropsychologia 1999;37:939-58.

19 Kapur N, Barker S, Burrows EH, et al. Herpes simplex encephalitis: long term magnetic resonance imaging and neuropsychological profile. $\mathcal{F}$ Neurol Neurosurg Psychiatry 1994;57:1334-42.

20 Jacobson RR, Lishman WA. Cortical and diencephalic lesions in Korsakoff's syndrome: a clinical and CT scan study. Psychol Med 1990;20:63-75.

21 Shimamura AP, Jernigan TL, Squire LR. Korsakoff's syndrome: radiological (CT) findings and neuropsychosyndrome: radiological (CT) findings and neur

22 Paller KA, Acharya A, Richardson BC, et al. Functional neuroimaging of cortical dysfunction in alcoholic Korsakoff's syndrome. F Cogn Neurosci 1997;9:277-93.

23 Kohler S, Black SE, Sinden M, et al. Memory impairments associated with hippocampal versus parahippocampalgyrus atrophy: an MR volumetry study in Alzheimer's disease. Neuropsychologia 1998;36:901-14

24 Jack CR, Twomey CK, Zinsmeister AR, et al. Anterior temporal lobes and hippocampal formations: normative volumetric measurements from MR images in young adults. Radiology 1989;172:549-54.

25 Watson C, Andermann F, Gloor P, et al. Anatomic basis of amygdaloid and hippocampal volume measurement by magnetic resonance imaging. Neurology 1992;42:1743-50.

26 Van Paesschen W, Duncan JS, Stevens JM, et al. Longitudinal quantitative hippocampal magnetic resonance imaging nal quantitative hippocampal magnetic resonance imaging study of adults with newly diagnosed partial

27 Cook MJ, Fish DR, Shorvon SD, et al. Hippocampal volumetric and morphometric studies in frontal and volumetric and morphometric studies in frontal

28 Ho SS, Kuzniecky RI, Gilliam F, et al. Congenital porencephaly: MR features and relationship to hippocampal sclerosis. Am f Neuroradiol 1998;19:135-41.

29 Bernasconi N, Bernasconi A, Andermann F, et al. Entorhinal cortex in temporal lobe epilepsy. Neurology 1999;52:870-87.

30 Juottonen K, Laakso MP, Partanen K, et al. Comparative MR analysis of the entorhinal cortex and hippocampus in diagnosis Alzheimer disease. Am $\mathcal{F}$ Neuroradiol 1999;20: 139-44.

31 Janowsky JS, Shimamura AP, Kritchevsky $\mathrm{M}$, et al. Cognitive impairment following frontal lobe damage and its relevance to human amnesia. Behav Neurosci 1989;103: 548-60.

32 Victor M, Adams RD, Collins GH. The Wernicke-Korsakoff syndrome 1st ed. Philadelphia: FA Davis, 1971.

33 Mayes AR, Meudell PR, Mann D, et al. Location of lesions in Korsakoff's syndrome: neuropsychological and neuropathological data on two patients. Cortex 1988;24:36788.

34 Fox NC, Warrington EK, Freeborough PA, et al. Presymptomatic hippocampal atrophy in Alzheimer's disease: a longitudinal MRI study. Brain 1996;119:2001-7.

35 Breier, A, Buchanan RW, Elkashef A, et al. Brain morphology and schizophrenia: a magnetic resonance imaging study of limbic, prefrontal cortex, and caudate structures. Arch Gen Psychiatry 1992;49:921-6.
36 Murphy DGM, DeCarli C, Schapiro MB, et al. Age-related differences in volumes of subcortical nuclei, brain matter, and cerebrospinal fluid in healthy men as measured with
magnetic resonance imaging. Arch Neurol 1992;49:839-45.

37 Murphy DGM, DeCarli CD, Daly E, et al. Volumetric magnetic resonance imaging in men with dementia of the Alzheimer type: correlations with disease severity. Biol Psychiatry 1993;34:612-21.

38 Bhatia S, Bookheimer SY, Gaillard WD, et al. Measurement of whole temporal lobe and hippocampus for MR volumetry: normative data. Neurology 1993;43:2006-10.

39 Aylward EH, Brandt J, Codori AM, et al. Reduced basal ganglia volume associated with the gene for Huntington's disease in asymptomatic at-risk persons. Neurology 1994; $44: 823-8$

40 Schlaepfer TE, Harris GJ, Tien AY, et al. Decreased regional cortical gray matter volume in schizophrenia. Am $f$ Psychiatry 1994;151:842-8.

41 Lehericy S, Baulac M, Chiras J, et al. Amygdalohippocampal MR volume measurement in the early stages of Alzheimer disease. Am f Neuroradiol 1994;15:927-37.

42 Howard RJ, Almeida O, Levy R, et al. Quantitative magnetic resonance imaging volumetry distinguishes delusional disorder from late-onset schizophrenia. Br 7 Psychiatry 1994; 165:474-80.

43 Bigler ED, Johnson SC, Anderson CV, et al. Traumatic brain injury and memory: the role of hippocampal atrophy. Neuropsychology 1996;10:333-42.

44 Pantel J, Schroder, Schad LR, et al. Quantitative magnetic resonance imaging and neuropsychological functions in dementia of the Alzheimer type. Psychol Med 1997;27:2129

45 Woodruff PWR, Wright IC, Shuriquie N, et al. Structural brain abnormalities in male schizophrenics reflect frontotemporal dissociation. Psychol Med 1997;27:1257-66.

46 Murphy DGM, DeCarli C, Daly E, et al. X-chromosome effects on female brain: a magnetic resonance imaging study of Turner's syndrome. Lancet 1993;342:1197-200.

47 Briellmann RS, Jackson GD, Kalnins R, et al. Hemicranial volume deficits in patients with temporal lobe epilepsy with and without hippocampal sclerosis. Epilepsia 1998;39: 1174-81.

48 Cheon J-E, Chang K-H, Kim HD, et al. MR of hippocampal sclerosis: comparison of qualitative and quantitative assessments. Am 7 Neuroradiol 1998;19:465-8.

49 Hashimoto M, Kitagaki H, Imamura T, et al. Medial tempo$\mathrm{ral}$ and whole-brain atrophy in dementia with Lewy bodies. Neurology 1998;51:357-62.

50 Laakso MP, Soinnen H, Partanen K, et al. MRI of the hippocampus in Alzheimer's disease: sensitivity, specificity, and analysis of the incorrectl classified subjects. Neurobiol Aging 1998;19:23-31.

51 Deicken RF, Pegues M, Amend D. Reduced hippocampal $\mathrm{N}$-acetylaspartate without volume loss in schizophrenia. Schizophr Res 1999;37:217-23.

52 Dickey CC, McCarley RW, Voglmaier MM, et al. Schizotypal personality disorder and MRI abnormalities of temporal lobe gray matter. Biol Psychiatry 1999;45:1393402 .

53 Warwick MM, Doody GA, Lawrie SM, et al. Volumetric magnetic resonance imaging study of the brain in subjects with sex chromosome aneuploidies. 7 Neurol Neurosurg Psychiatry 1999;66:628-32.

54 Laakso MP, Paranen K, Riekkinen Jr P, et al. Hippocampal volumes in Alzheimer's disease, Parkinson's disease with and without dementia, and in vascular dementia: an MRI study. Neurology 1996;46:678-81.

55 Kuzniecky R, Hugg JW, Hetherington H, et al. Relative utility of ${ }^{1} \mathrm{H}$ spectroscopic imaging and hippocampal volumetry in the lateralization of mesial temporal lobe epilepsy. Neurology 1998;51:66-71.

56 Woermann FG, Barker GJ, Birnie KD, et al. Regional changes in hippocampal T2 relaxation and volume: a quantitative magnetic resonance imaging study of hippocampal sclerosis. F Neurol Neurosurg Psychiatry 1998;66: 656-64.

57 Golebiowski M, Barcikowska M, Pfeffer A. Magnetic resonance imaging-based hippocampal volumetry in patients with dementia of the Alzheimer type. Dement Geriatr Cogn Disord 1999;10:284-8.

58 Kuzniecky R, Bilir E, Gilliam F, et al. Quantitative MRI in temporal lobe epilepsy: evidence for fornix atrophy. Neurology 1999;53:496-501.

59 Maguire EA, Gadian DG, Johnsrude IS, et al. Navigationrelated structural change in the hippocampi of taxi drivers. Proc Natl Acad Sci USA 2000;97:4398-403. 